\title{
Survey of U.S. Biomechanics Instruction
}

\section{Prof. Anton E Bowden, Brigham Young University}

Anton E. Bowden is an Associate Professor of Mechanical Engineering and director of the BYU Applied Biomechanics Engineering Laboratory at Brigham Young University. His background and research interests are in spinal biomechanics, biomedical device design, computational biomechanics, and recently in engineering education. He received his $\mathrm{PhD}$ in Bioengineering from University of Utah and his BS in Mechanical Engineering from Utah State University. He is a licensed professional engineer and a recipient of a National Science Foundation CAREER Award. He is grateful to have been awarded the Weidman Professorship in Leadership and to have recently been recognized with BYU's Technology Transfer Award.

\section{Dr. Ruth Ochia P.E., Temple University}

Ruth S. Ochia received the B.S. degree in biomedical engineering from The Johns Hopkins University, Baltimore, MD, in 1992 and the Ph.D. degree in bioengineering from the University of Washington, Seattle, WA, in 2000. From 2000 to 2002, she was a Post-doctoral Fellow in the Center of Locomotion Studies, at The Pennsylvania State University, State College, PA. From 2002 to 2006, she was a Postdoctoral Fellow and then Assistant Professor at Rush University Medical Center, Chicago, IL. From 2006 to 2009, she was a Senior Associate with Exponent, Inc. From 2009 to 2013, she was principal of RSO Consulting, LLC, and taught as an Adjunct Professor at Widener University, Chester, PA. Since 2013, she has been an Associate Professor with the Bioengineering Department, Temple University, Philadelphia, PA. Her research interests have included biomechanics, primarily focusing on spine-related injuries and degeneration. Currently, her interests are in engineering education, curriculum development and assessment. Dr. Ochia is a licensed professional engineer in the state of Pennsylvania. She is a member of the Orthopaedic Research Society (ORS), American Society of Mechanical Engineers (ASME), American Society for Engineering Education (ASEE), and Biomedical Engineering Society (BMES).

\section{Dr. Dennis Lee Eggett, Depsrtment of Statistics - Brigham Young University}

Dr. Eggett received his BS and MS in Statistics from BYU and his PhD in Applied Statistics from North Carolina State University. He worked in industry for ten years at Pacific Northwest National Laboratory. Since 1997 Dr. Eggett has been the director of the Center for Statistical Consultation and Collaborative Research in the Department of Statistics at Brigham Young University. His specialties include linear models and mixed model analysis. 


\title{
Survey of U.S. Biomechanics Instruction
}

\begin{abstract}
This paper presents the results of a web survey sent to all known U.S. engineering instructors who are currently or have recently taught a biomechanics course. Survey questions addressed: Course (student) demographics, instructor experience, course structure, resource material (including textbooks), satisfaction with course material, and topics covered within the biomechanics course. Results indicate that there is tremendous diversity in terms of primary course material used to teach engineering biomechanics, with over $23 \%$ of respondents not using any formal textbook at all (i.e., using custom course material) and $83 \%$ of the respondents augmenting their primary textbook with additional material.

Topics from Bone Mechanics, Soft Tissue Mechanics, Tissue Remodeling, and Orthopaedic Biomechanics were most highly represented among current biomechanics courses. Biofluid Mechanics, Vascular Mechanics, Respiratory Mechanics, Medical Imaging \& Mechanics, Injury Biomechanics, and Biomedical Engineering Ethics were least represented. There was a significant statistical correlation between the current research topics of the respondents and the included topics in their engineering biomechanics courses. In an open-response query in the survey, respondents identified three additional resources that would be most helpful in teaching their current biomechanics course: 1) a "better," more unified textbook, 2) "meaningful" and descriptive laboratory experiments that can be accomplished using routine equipment, and 3 ) sample and homework problems.

This study represents preliminary work identifying the "state of the practice" in terms of engineering biomechanics education. We anticipate that the results will provide discussion points among biomechanics educators and hopefully lead to collaborative efforts to develop a more unified curriculum
\end{abstract}

\section{Introduction}

Although pioneering work in biomechanics dates back centuries, formal biomechanics instruction has only been a part of modem university education for the last several decades. Indeed, most biomechanics courses have arisen ad hoc based on the research experiences of a single faculty member. Anecdotally, teaching methods for biomechanics courses vary substantially based on institution. This concept is supported by a Whitaker Foundation survey concerning biomechanics instruction in the United States ${ }^{1}$. The study indicated that that at that time, the core topics in biomechanics instruction were statics, dynamics, strength of materials, and biotransport phenomena. It was suggested that imaging and computational biomechanics should be included with this "core" of biomechanics. This study also indicated that there was a developing paradigm shift in curricula towards including more cellular and molecular based topics. In general, the biomechanics instruction at U.S. institutions at the time was mainly based on faculty interests and composition. Since that time, the literature concerning biomechanics 
instruction has been largely with the development of pedagogical tools for active learning and student engagement within particular courses ${ }^{2-4}$. It is of interest to the engineering education community to identify common practices, instructional techniques, and course materials that are currently being used. This knowledge may lead to identification of gaps in available resource materials that could be filled through collaborative efforts by the biomechanics community.

This objective of the present work was to identify the current "state of the practice" in terms of undergraduate engineering biomechanics education.

\section{Methods}

The proposed research utilized a web survey (Qualtrics survey) to query all known US engineering instructors who are currently or have recently taught an engineering biomechanics course, which is a course taught as part of an engineering department curriculum. Instructor contact information was obtained based on openly available course information gleaned from U.S. University websites. Additionally, the survey link was posted to the e-mail group listservers for the biomedical engineering section of the American Society of Engineering Educators (ASEE), as well as, the Biomch-L e-mail listserver. From these sources, we identified approximately 90 faculty members that were included in the e-mail solicitation to participate in the proposed research.

The survey questions addressed course (student) demographics, instructor experience, course structure, resource material (including textbooks), satisfaction with course material, and topics covered within the course. No identifying information was collected. The study was classified as "exempt" following review by our Institutional Review Board.

The levels of the different categories, times taught, class level, etc., were compared using Analysis of variance (ANOVA). Following the ANOVA a post-hoc Tukey test was performed to compare pairwise differences in the levels of the particular variable of interest. Kendall's Tau Rank Correlation test was used to determine correlations between research areas and course topics included in biomechanics courses. The dependent variables used in the analysis were the Likert scale variables relative to inclusion of the topic of interest. The significance level for all analyses was set at 0.05 .

\section{Results}

\section{Instructor Experience and Demographics}

We received 48 responses from 44 unique IP addresses, of which 35 respondents fully completed the survey. The average response time for the survey was 15 minutes with a median response time of 12 minutes. Of the respondents, $76 \%$ had taught their biomechanics class at least 2 times, with $44 \%$ having taught their biomechanics over 5 times. Over $85 \%$ of the respondents 
indicated that conducting research was part of their professional responsibilities. The research topics of these instructors are indicated in Figure 1.

\section{Student demographics}

Survey respondents were asked to provide basic demographical information on the students in their biomechanics class. Based on their responses, only a small fraction of biomechanics classes included Freshmen (3\%) or Sophomores (22\%). Most of biomechanics education is reaching upper level undergraduate students (46\% of classes included Juniors, $55 \%$ included Seniors) with first year graduate students comprising the next largest population (38\% of classes). A large majority (70\%) of instructor respondents indicated that their biomechanics course was a required part of the curriculum as opposed to an elective.

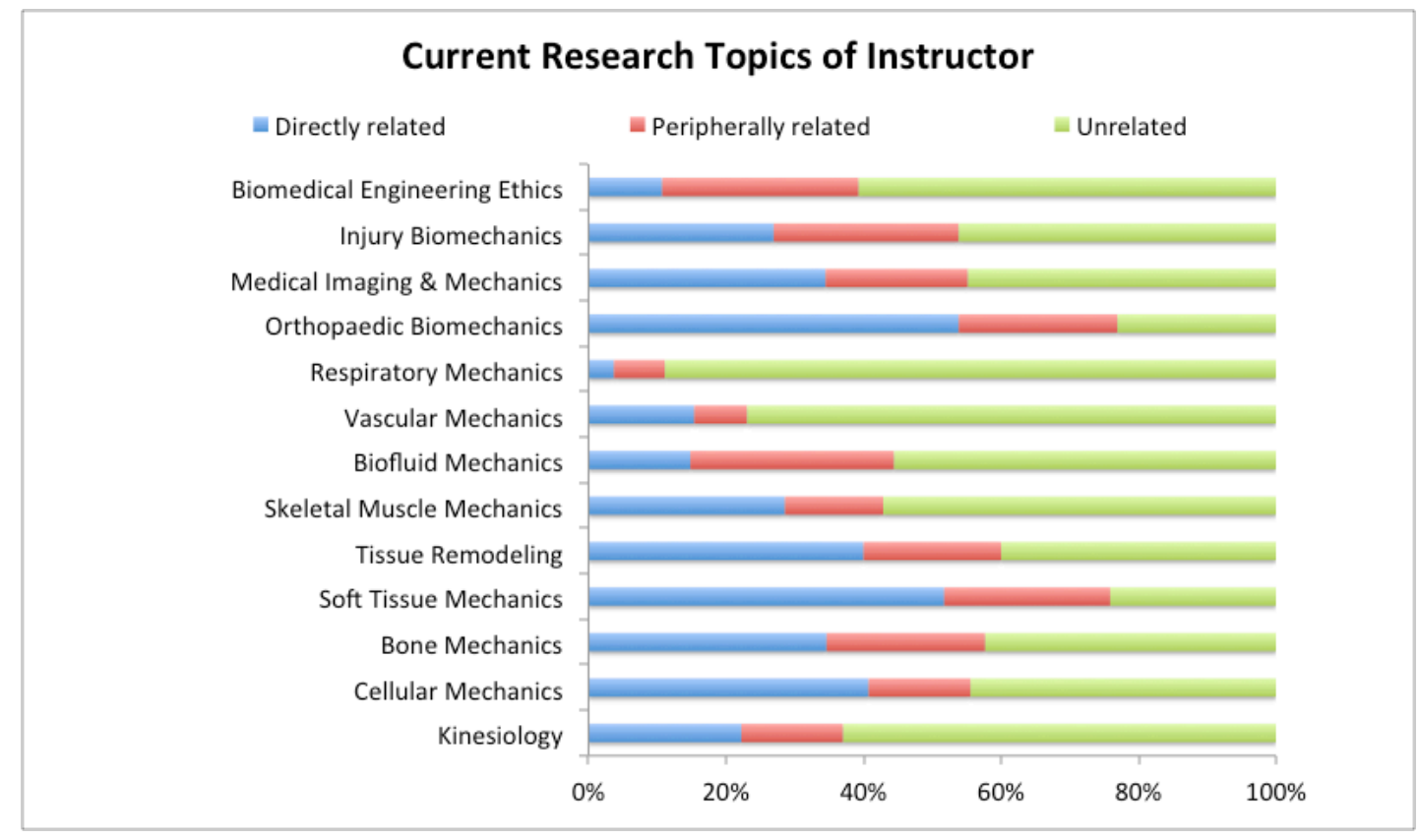

Figure 1. Research topics of biomechanics instructors

The survey was specifically targeted towards engineering educators (i.e., biomechanics courses being taught from engineering departments). Thus, it was not surprising that the composition of the instructor respondents' classes was primarily engineers. 35\% of classes included Mechanical Engineering students, 84\% included Biomedical Engineering students, 11\% included Exercise Science students, and 5\% included students from other majors besides those listed above. 


\section{Course Structure}

The majority of the respondents (58\%) stated that the average enrollment in their biomechanics class was over 41 students in size. Only $22 \%$ of respondents indicated that their average class size was less than 20 students. Over $83 \%$ of biomechanics classes were categorized as having 3 in-class lecture hours per week. Most respondents (69\%) indicated that their students had some kind of biomechanics laboratory experience. However, the cumulative laboratory hours associated with biomechanics instruction was relatively modest (Figure 2).

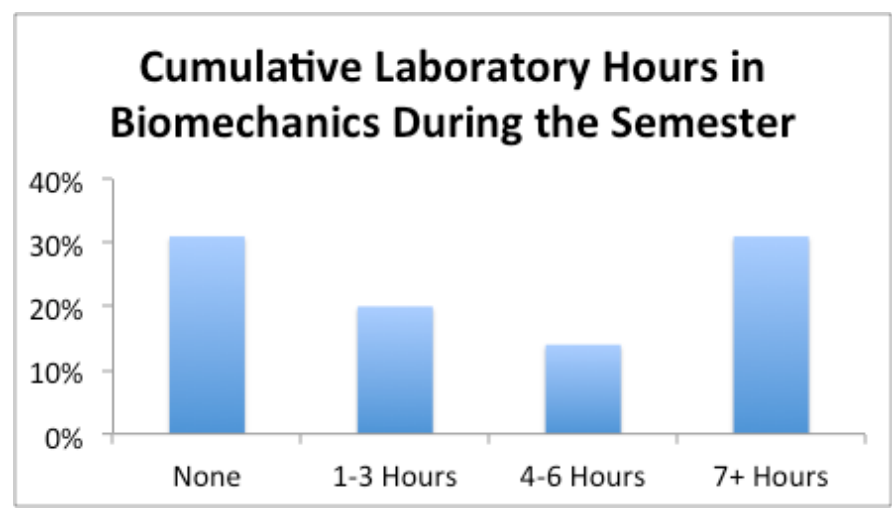

Figure 2. Instructor reported laboratory hours in biomechanics during the semester. Note that this includes both laboratory hours directly associated with the biomechanics class, as well as laboratory hours required by the curriculum but not directly associated with the class.

\section{Textbook}

There was no clear consensus on a primary textbook used in biomechanics courses. The most popular textbook was only used by $11 \%$ of respondents. $83 \%$ of respondents either required more than one textbook or used custom course materials (journal articles, custom PowerPoints, videos, software, etc.) to supplement their primary textbook. Custom course materials play a significant role in biomechanics education. Over $23 \%$ of respondents used custom course material as their primary "textbook" and an additional $33 \%$ used custom course material to supplement their course.

Most of the respondents were unsatisfied with the textbooks available for biomechanics (Figure 3). None of the mean scores for any textbook category exceeded a "Neutral" rating (4.0) on a 7 point scale between "Extremely dissatisfied" (1.0) and "Extremely satisfied" (7.0). Based on the instructor responses, the available texts have a low cost/benefit ratio for students, do not contain helpful homework problems, software examples, or laboratory exercises, and do not adequately cover the topics that the instructor would like to teach. The highest mean score for textbook was in "Appropriate writing level for the class you teach", which averaged a 3.71/7.0 (stdev 1.64). The lowest mean score for textbook was in "Helpful laboratory exercises", which averaged a 1.56/7.0 (stdev 1.59). 


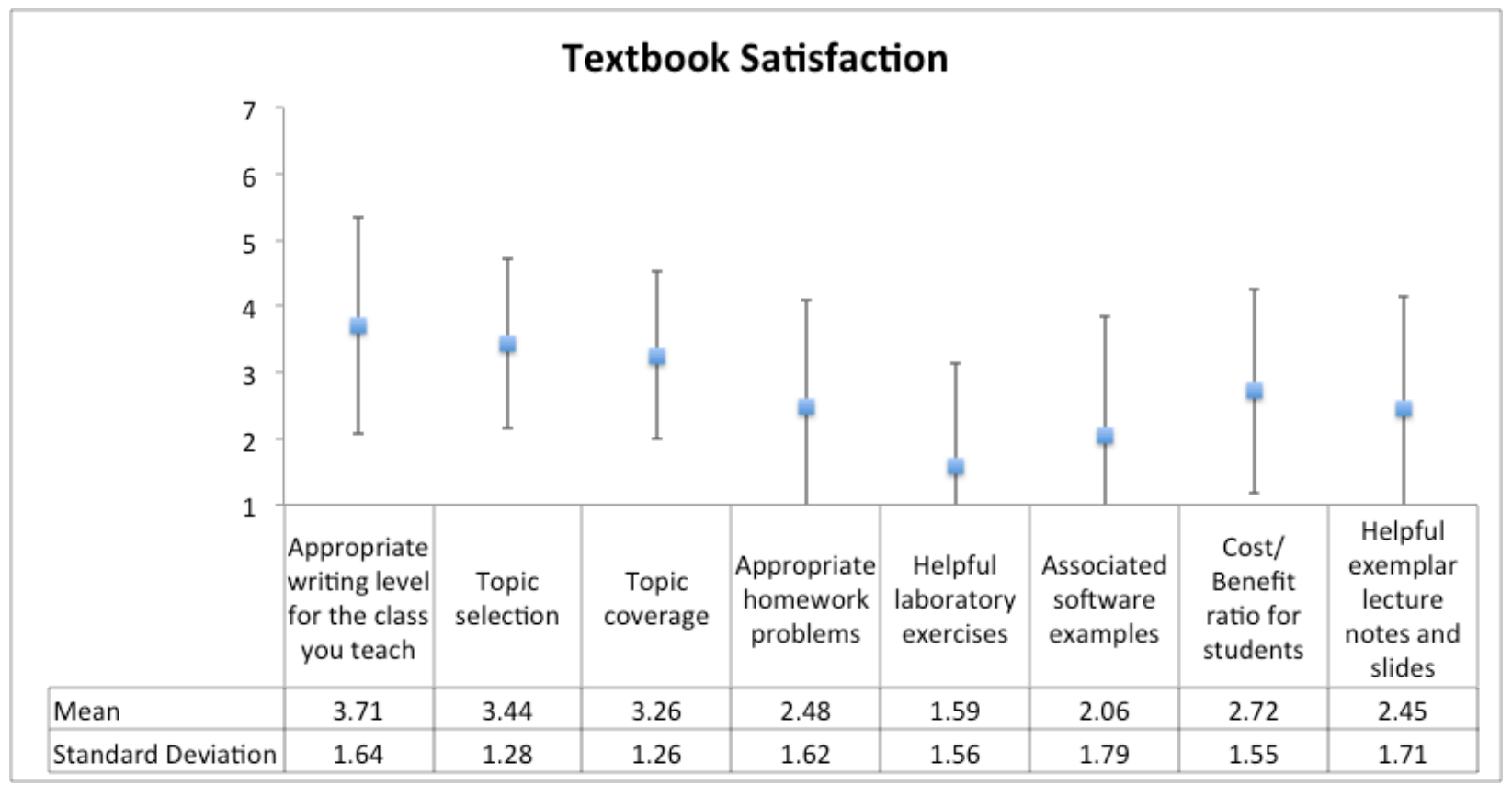

Figure 3: Responses to textbook satisfaction where 7 represents "extremely satisfied" and 1 represents "extremely dissatisfied." A rating of 4.0 represents a "Neutral" rating.

\section{Course Topic Coverage}

Topics from Soft Tissue Mechanics (Figure 4), Orthopaedic Biomechanics, Bone Mechanics, and Tissue Remodeling were most highly represented among current biomechanics courses, with each receiving an inclusion rate of over $80 \%$. In particular, some specific subtopics, such as viscoelasticity, were included in over $90 \%$ of the surveyed courses (see Appendix). In contrast, Biofluid Mechanics, Vascular Mechanics, Respiratory Mechanics, Medical Imaging \& Mechanics, Injury Biomechanics, and Biomedical Engineering Ethics were least represented, with each receiving less than a $30 \%$ inclusion rate. Complete data regarding subtopic coverage are included in the Appendix. 


\section{Biomechanics Course Topics Coverage}

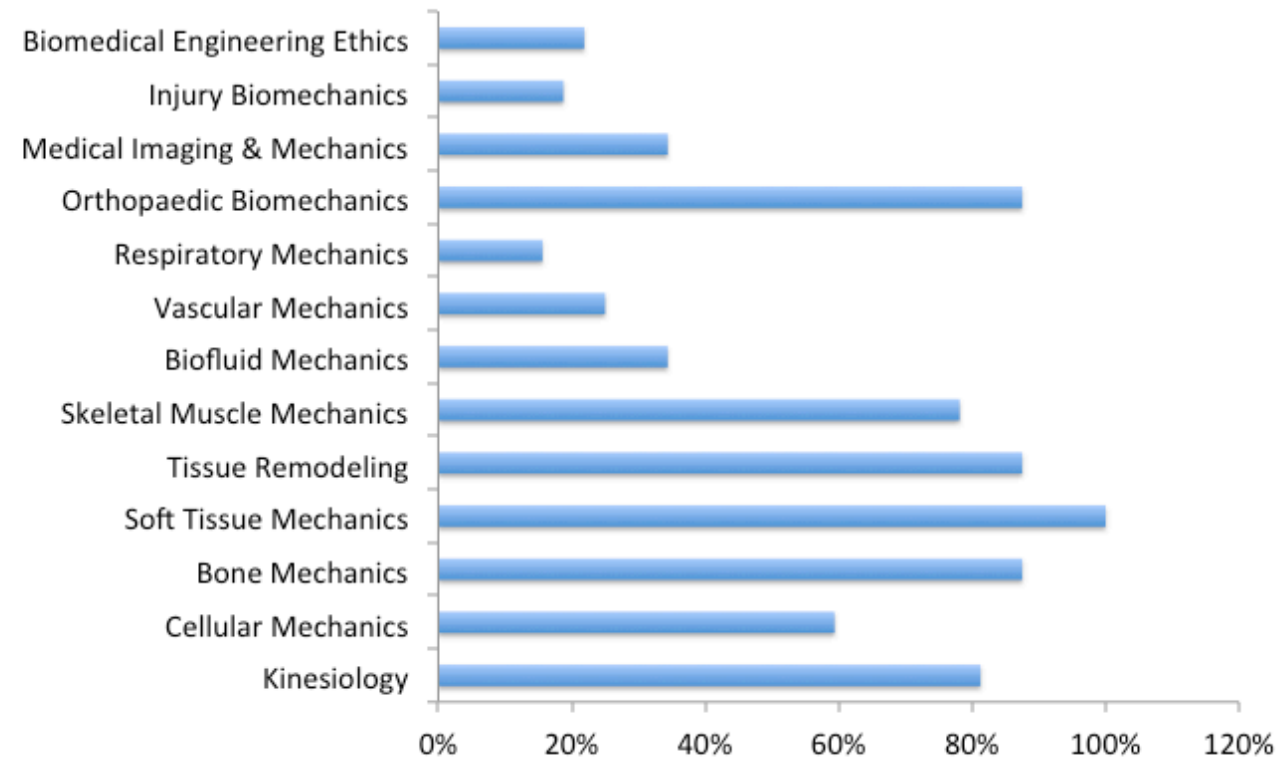

Figure 4. Biomechanics course topic coverage as reported by the instructor respondents. A topic was considered to be included if any of the subtopics were indicated to be included by the instructor (see Appendix for more detailed information on subtopic inclusion).

Several topics covered in the courses were, as expected, dependent on the level/department of the students enrolled within the class, i.e. Graduate students versus Lower Division Undergraduates or bioengineering students versus exercise science students (Table 1). We did not specifically exclude doctoral level classes, and respondents indicated that "advanced undergraduate students" were included in $14 \%$ of courses. However, only 1 respondent indicated that their course was comprised exclusively of doctoral level students, thus our statistical analysis lumped all graduate courses together. Similarly, we lumped lower division undergraduates (Freshmen, Sophomores) together and upper division undergraduates together (Juniors, Seniors) to increase the resolving power of our statistical analysis. The p-values in Table 1 demonstrate the statistically significant results from our analysis. For example, Soft Tissue Mechanics course materials were common in graduate classes, but rarely presented to lower division undergraduate students.

There was a significant correlation between the current research of the respondents and the included topics in their engineering biomechanics courses (Kendall's Tau Rank Correlation test, $\mathrm{p}=0.0088$ ); for example, compare Figures 1 and 4. Many respondents directly or peripherally do research in bone mechanics (58\%) or have had training in this topic (47\%) and include bone mechanics topics in their biomechanics courses (88\%). Statistically significant deviations from the correlation are highlighted in Table 2. Notable outliers were seen in the areas of kinesiology 
( $81 \%$ inclusion rate, but only a $33 \%$ current research or $28 \%$ training research relationship), skeletal mechanics (78\% inclusion rate, but only a $31 \%$ research relationship), and Medical Imaging \& Mechanics (34\% inclusion rate, with a 56\% research relationship).

Table 1: Correlations of course topics based on student class level and department. Statistically significant p values are shown after student class level or departmental differences, “-“" indicates no significant differences found among these groups. GRD - graduate students, UPD - upper division undergraduate students (Junior/Senior), LWD -lower division undergraduate students (Freshmen/Sophomore), BIO - bioengineering department, EXSC - exercise science department, and $\mathrm{MECH}$ - mechanical engineering department.

\begin{tabular}{ccc}
\hline & Class Level & Student Department \\
\hline Kinesiology & - & BIO vs EXSC, 0.0269 \\
Cellular Biomechanics & - & - \\
Bone Biomechanics & - & - \\
Soft Tissue Biomechanics & GRD vs LWD, 0.0143 & - \\
Tissue Remodeling & GRD vs LWD, 0.0025 & - \\
Muscle Mechanics & UPD vs GRD, 0.0094 & BIO vs EXSC, 0.0231 \\
Biofluid Mechanics & - & BIO vs EXSC, 0.0444 \\
Vascular Mechanics & - & BIO vs MECH, 0.0456 \\
Respiratory Mechanics & - & - \\
Orthopaedic Biomechanics & - & - \\
Medical Imaging \& Mechanics & - & - \\
Injury Biomechanics & - & - \\
Biomedical Engineering Ethics & - & \\
\hline
\end{tabular}


Table 2: Comparisons between instructor research and prior training with topics covered in course. Statistically significant $p$-values are given in the table indicating statistically significant likelihood that the indicated topic covered in the biomechanics course is not directly related to current instructor research or prior instructor research training, "_" indicates that the topics overlapped.

\begin{tabular}{ccc}
\hline & Current Research & Training Research \\
\hline Kinesiology & 0.0005 & 0.0015 \\
Cellular Biomechanics & 0.0166 & 0.0293 \\
Bone Biomechanics & - & - \\
Soft Tissue Biomechanics & 0.0064 & 0.0018 \\
Tissue Remodeling & - & - \\
Muscle Mechanics & 0.0318 & - \\
Biofluid Mechanics & - & - \\
Vascular Mechanics & - & - \\
Respiratory Mechanics & - & 0.0009 \\
Orthopaedic Biomechanics & 0.0091 & - \\
Medical Imaging \& Mechanics & - & 0.012 \\
Injury Biomechanics & - & - \\
Biomedical Engineering Ethics & 0.0005 & - \\
\hline
\end{tabular}

\section{Additional Insights}

There was a large variation between the course topic coverage between respondents. Six core topics were covered in over $80 \%$ of biomechanics courses (Orthopaedic Biomechanics, Skeletal Muscle Mechanics, Tissue Remodeling, Soft Tissue Biomechanics, Bone Mechanics, and Kinesiology). However, based on a cluster analysis of the subtopics within those core topics, there was no consistent group of subtopics that were consistently chosen by instructors for inclusion in biomechanics course curriculum.

There was an $80 \%$ response rate to the open-response question, "In your opinion, what additional resources (if any) would be most helpful to you in teaching your biomechanics course?" The highest frequency responses were unexpectedly easy to categorize as: 1) a "better," more unified textbook $(43 \%), 2)$ "meaningful" and descriptive laboratory experiments that can be accomplished using routine equipment (29\%), and 3) sample and homework problems (21\%).

\section{Discussion}

The current study reviewed responses from 37 instructor respondents regarding current biomechanics courses taught in engineering departments in the United States. One major finding showed that instructors are not happy with the available textbooks available and thus heavily supplement with other course materials or forgo the use of a textbook entirely. There was tremendous diversity in terms of primary course material used to teach engineering 
biomechanics, with over $23 \%$ of respondents not using any formal textbook at all (i.e., using custom course material) and $33 \%$ of the respondents using basic mechanics textbooks that contain no biomechanical content. A large majority $(78 \%)$ of respondents also indicated that they

utilized secondary course material consisting of selections from other textbooks and articles from the primary literature. However, the topics, intended audience, and associated content (homework problems, lab exercises, and available lecture materials) vary widely and from personal experience are insufficient for building an effective class. Our personal experience, combined with anecdotal conversations with other engineering biomechanics instructors, leads us to believe that faculty use an ad hoc combination of textbooks, course packets, and personally developed (but not disseminated) lecture notes, lecture slides, and homework problems to teach from. Lab experiences tend to be "homegrown", but provide substantial benefit for students that could potentially be increased through sharing of ideas.

Core topics covered in current biomechanics instruction include bone, soft tissue, muscle and orthopaedic mechanics, with additional coverage of kinesiology and tissue remodeling topics. These topics are very similar to those described in the Whitaker Foundation study from 2000, which illustrated that "core" topics were based on statics, dynamics, and strength of materials concepts. In contrast, the Whitaker Study reported that biotransport phenomena were a major topic of study, which was not seen in the current study, (e.g., biofluids, vascular mechanics, and respiratory mechanics were not well covered in current biomechanics curricula). There was general inclusion of cellular mechanics topics, such as cellular architecture $(60 \%)$ and constitutive properties ( $45 \%$ ) as was predicted by the Whitaker study. However medical imaging topics remained largely excluded from the current biomechanics courses. The current study did not address whether particular topics, such as biotransport phenomena, are covered in other areas within a general bioengineering curricula and are less likely to be included in biomechanics courses.

A conversation among those instructors who teach biomechanics courses within the U.S. is needed. Discussion of a general range of topics to be covered within biomechanics that broadly reflects both fundamental topics and current research areas is needed. In addition, formal conversations with industry regarding biomechanics knowledge use in the workplace would be beneficial in terms of identifying their needs with regards to biomechanics instruction.

\section{Bibliography}

1. Hung, C., Sah, R., 2000. Whitaker Foundation Summit 2000: Report on Biomechanics Curriculum.

2. Pandy, M.G., Petrosino, A.J., Austin, B.A., Barr, R.E., 2004. Assessing Adaptive Expertise in Undergraduate Biomechanics. Journal of Engineering Education 93, 211-222.

3. Panta, Y.M., Butcher, M., 2012. Development of an Integrative Biomechanics Course for STEM Majors, in: ASEE (Ed.), ASEE Annual Conference. ASEE, San Antonio, TX. 4. Roselli, R.J., Brophy, S.P., 2013. Effectiveness of Challenge-Based Instruction in Biomechanics. Journal of Engineering Education 95, 311-324. 
Appendix: Subtopic Course Coverage as Reported by Biomechanics Instructors
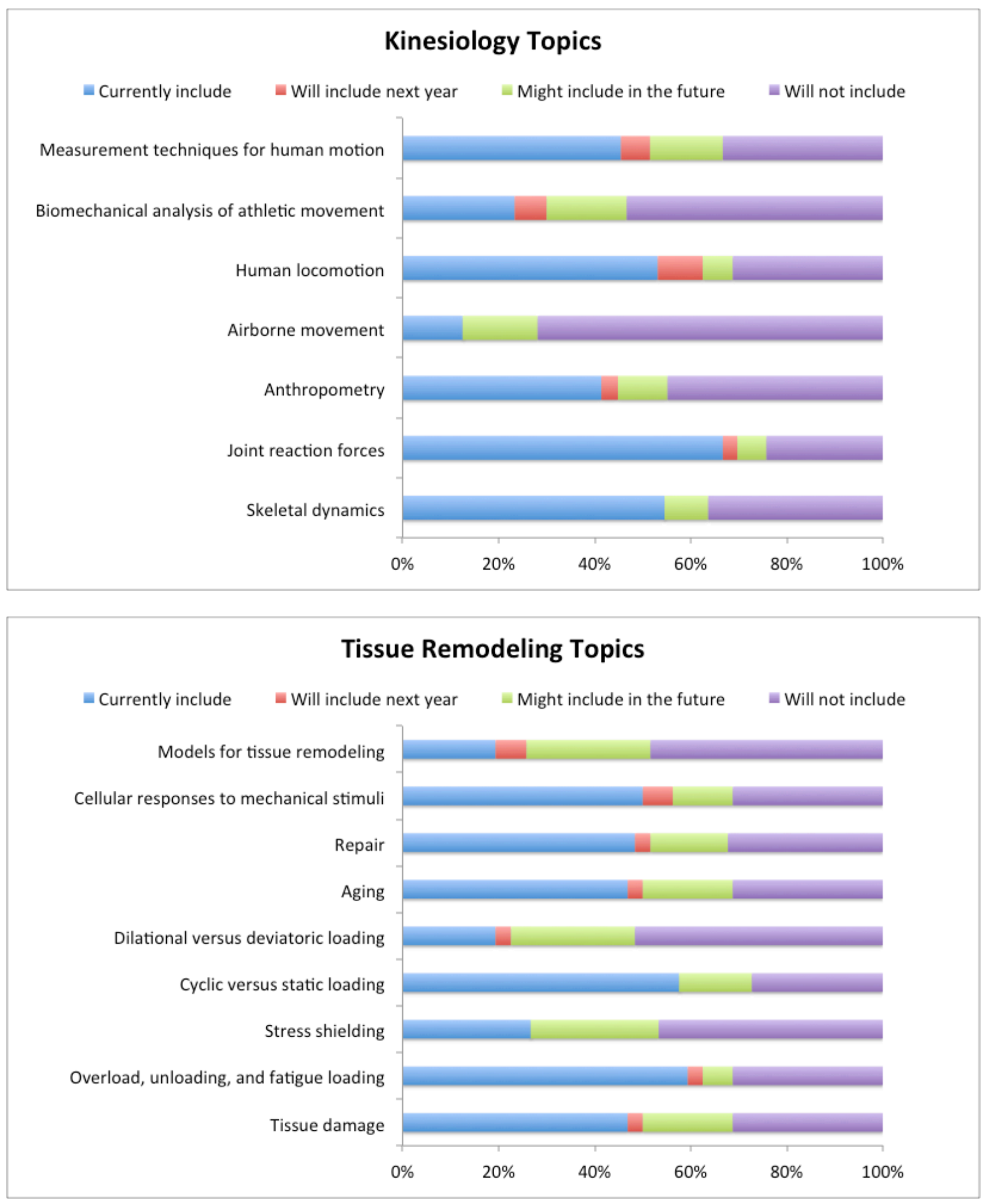


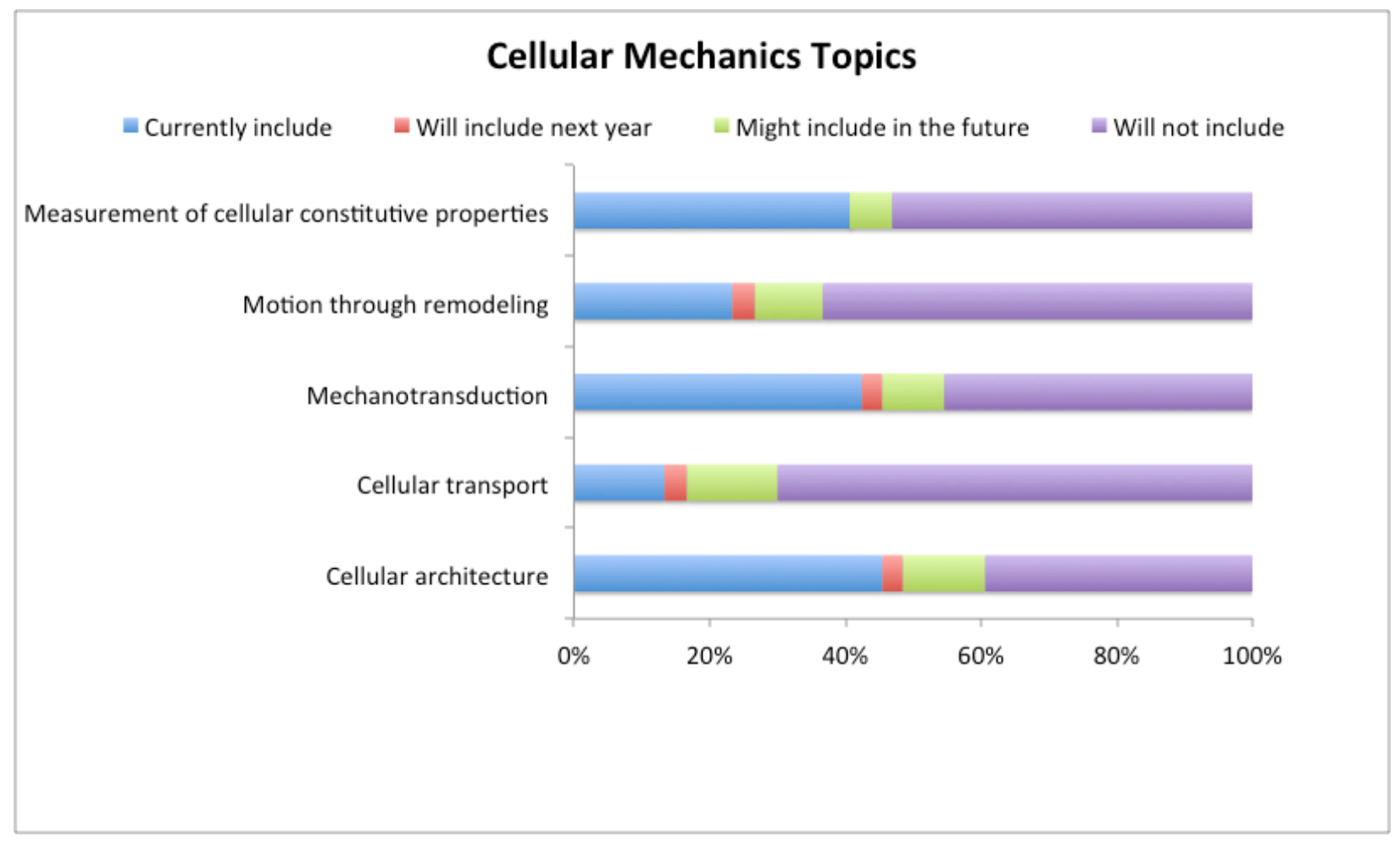

\section{Bone Mechanics Topics}
Currently include
Will include next year
Might include in the future
Will not include

Materials testing of hard tissues

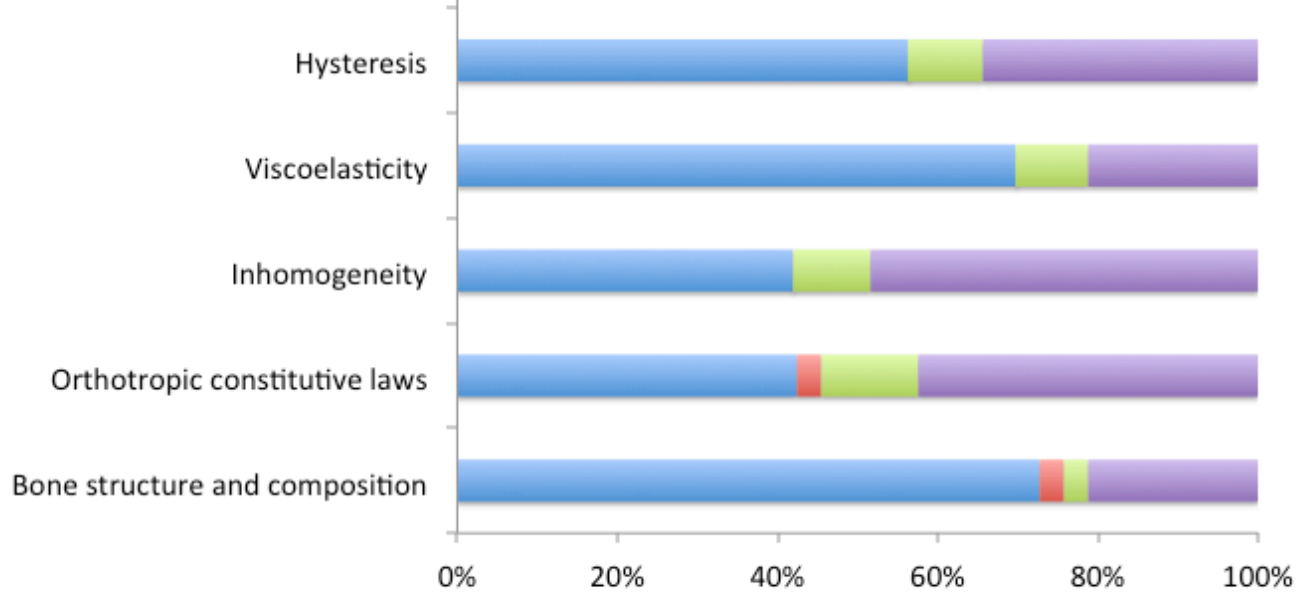



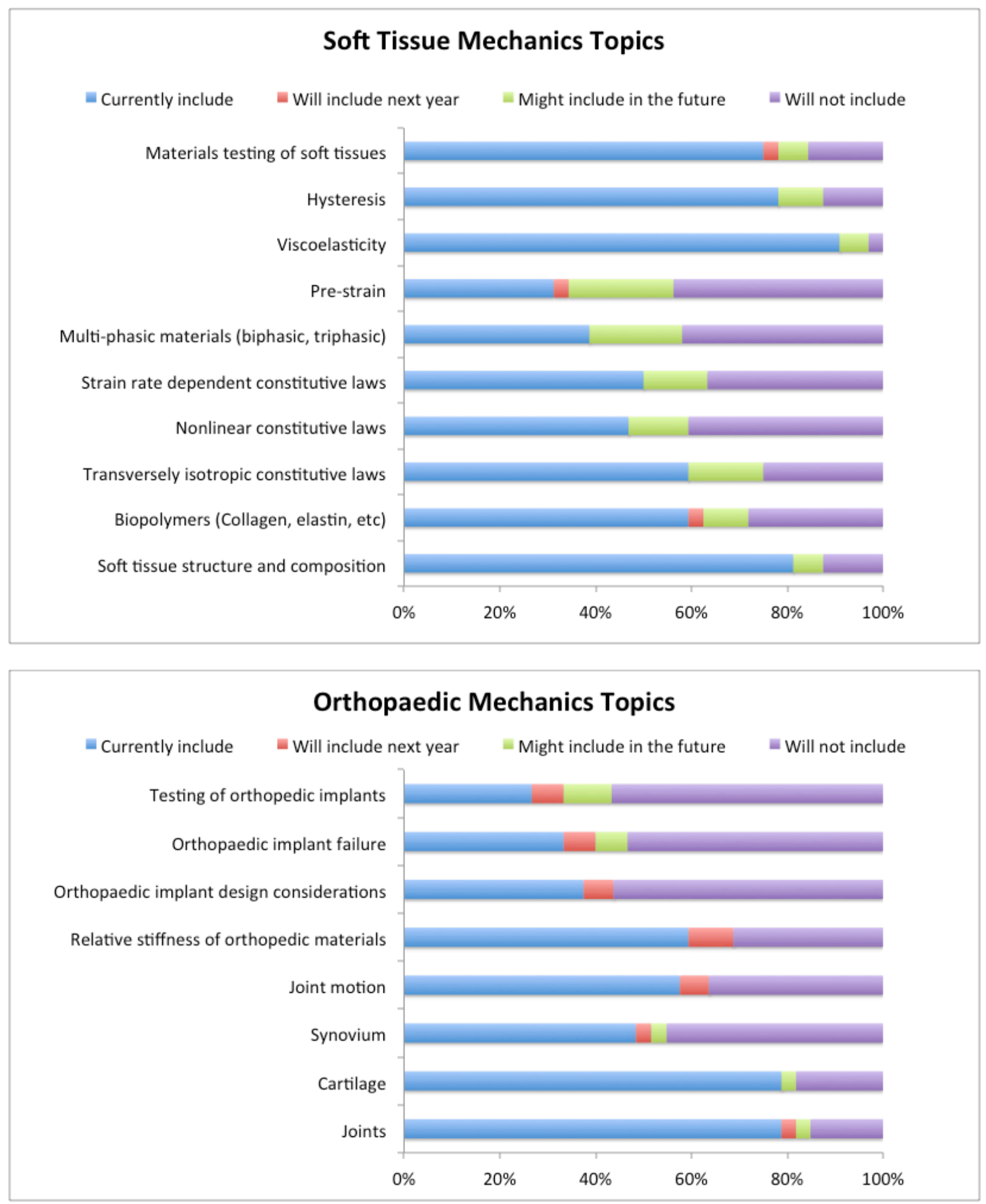

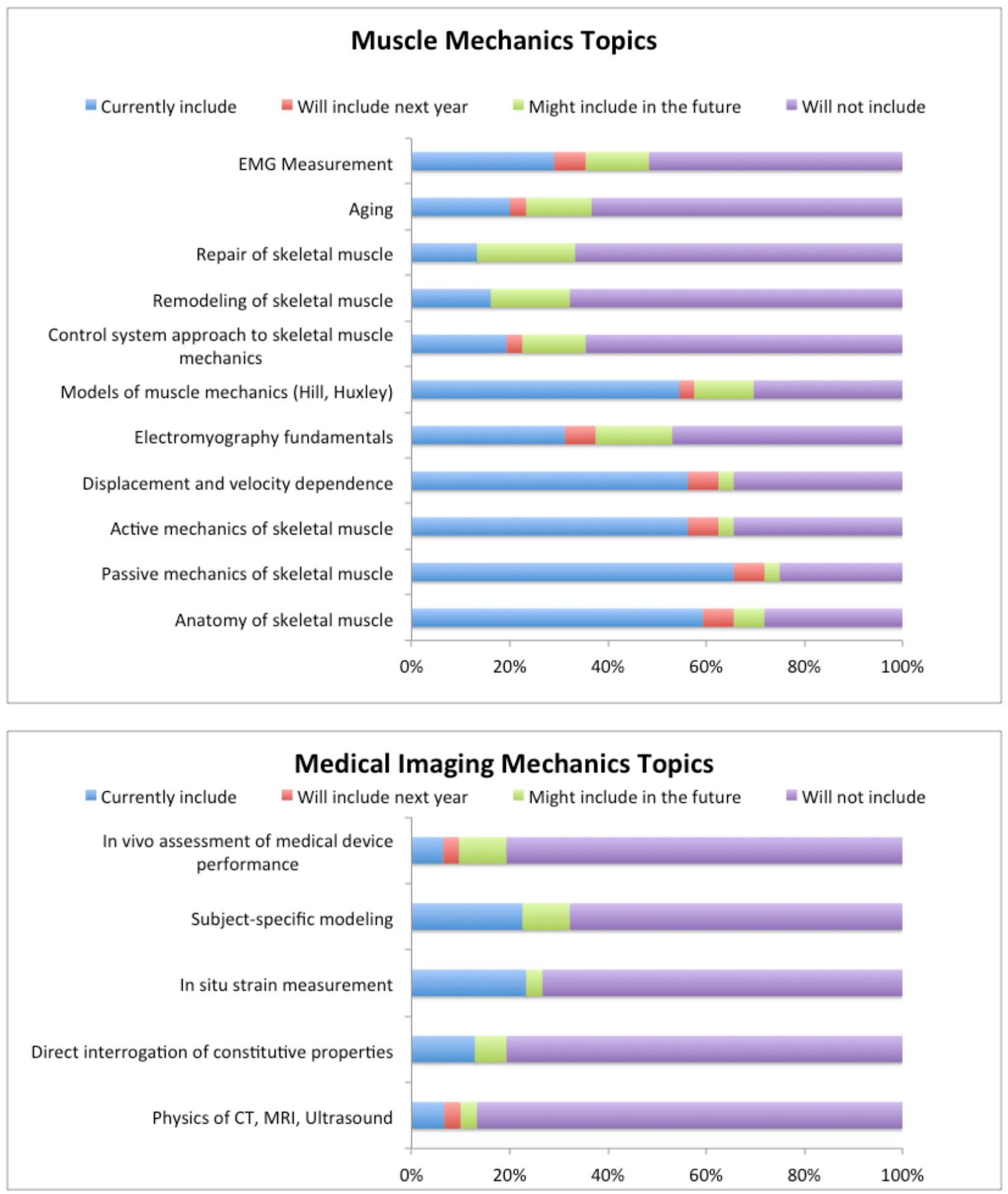


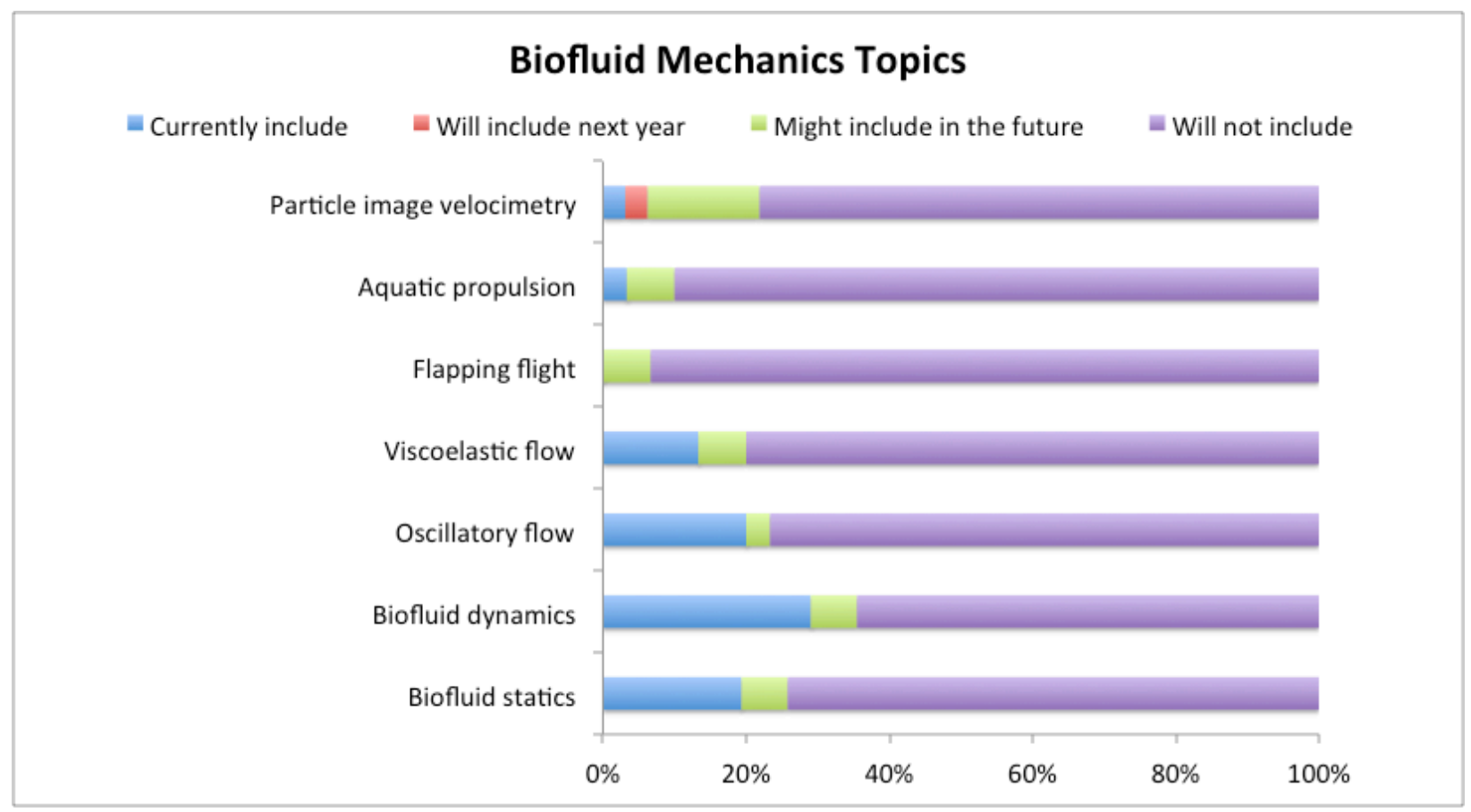

\section{Vascular Mechanics Topics}

- Currently include Will include next year $\quad$ Might include in the future Will not include

In vivo blood flow measurement

Stents and vascular grafts

Aneurisms

Vascular disease

Vascular bifurcations

Pulsatile flow

Arterial smooth muscle

Fluid-structure interactions

Shear thinning

Blood composition

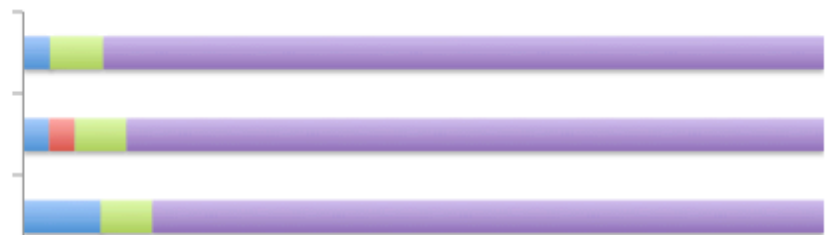

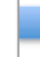
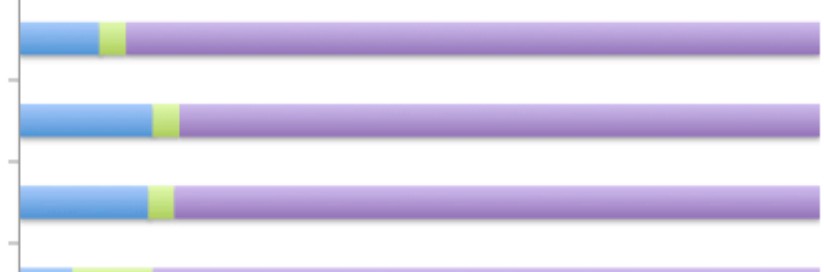

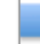

\footnotetext{
(1)
}

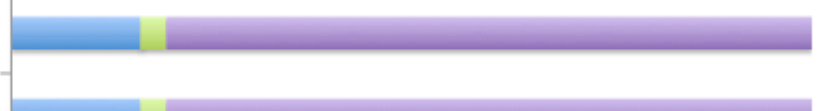

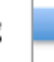

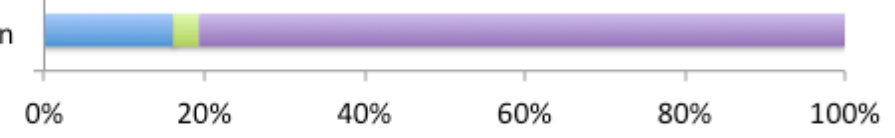




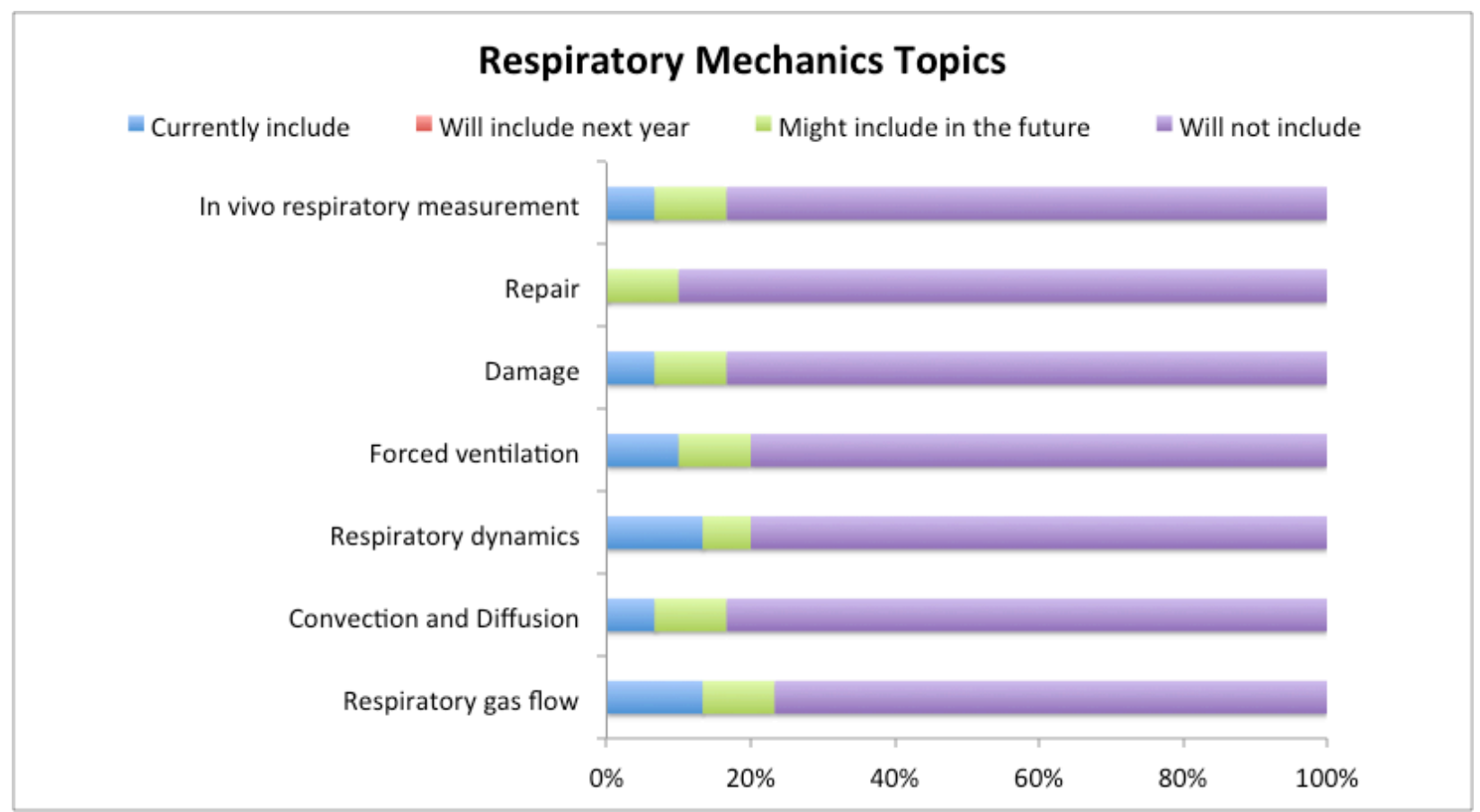

\section{Injury Mechanics Topics}
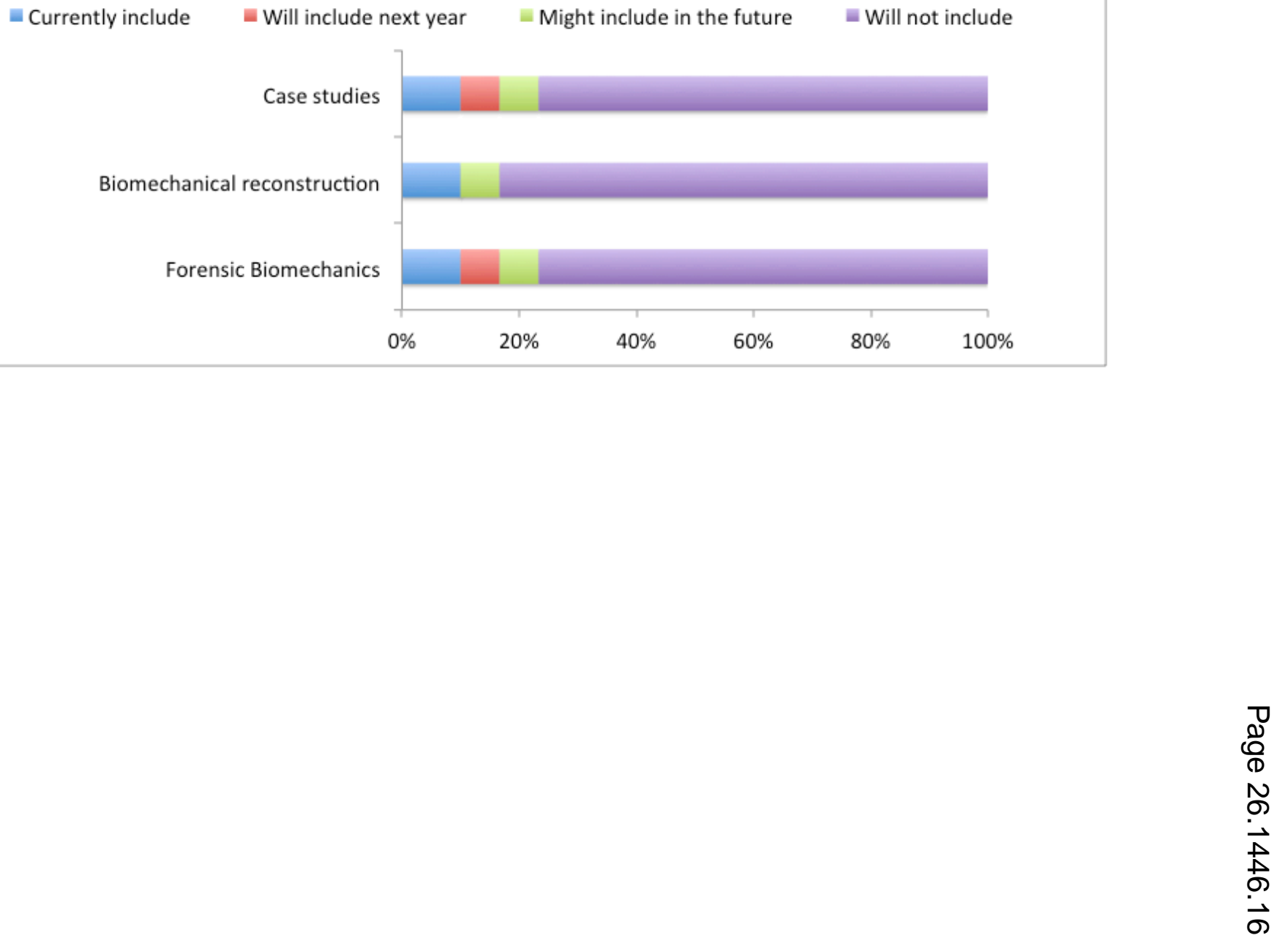